\title{
Stress urinary incontinence in women and lower urinary tract symptoms in men: What are the remaining questions?
}

\author{
Sender Herschorn, MD, FRCSC \\ Professor Division of Urology, University of Toronto, and Head of Urodynamics Laboratory, Sunnybrook Health Sciences Centre, Toronto, Ontario
}

Cite as: Can Urol Assoc J 2012;6(5):S148. http://dx.doi.org/10.5489/cuaj.12230

T he educational sessions of the 2012 Canadian Urology Forum were informative not only because of the evidencebased and practical guidance they provided, but also due to the gaps in knowledge identified during these comprehensive overviews. The following brief review provides a recap of the most important of these outstanding questions, whose answers would provide invaluable information in helping health-care professionals manage stress urinary incontinence (SUI) in women and lower urinary tract symptoms (LUTS) in men.

\section{SUI}

One of the gaps in the knowledge that needs to be closed is the absence of evidence to guide a structured workup of women with SUI. Urodynamic testing in particular still requires objectively derived evidence to direct its use. Although there are good data regarding the incidence and management of complications of midurethral slings (MUS), there are some remaining areas of controversy. For example, the optimal method of addressing obstructed voiding in a patient post-MUS has not been adequately resolved. Another area where the lack of agreement in the literature was striking was in how to define success of an intervention for SUI. What is apparent is that a multidimensional approach is necessary. Future efforts to standardize this assessment should take into account patient-focused outcomes as well as dry rates. Furthermore, should a MUS failure be identified, there is very little evidence guiding clinicians in recommending next steps, specifically regarding subsequent surgical procedures.

\section{LUTS}

In the treatment of benign prostatic hyperplasia $(\mathrm{BPH})$, there are several widely utilized medical therapies such as alpha-blockers and 5-alpha reductase inhibitors and occasionally antimuscarinics that have become standard therapies in the clinical practice guidelines. However, there is now another class of medications with excellent evidence (the PDE-5 inhibitors) and their place in treatment algorithms relative to the existing recommended medications will have to be decided.

Among men whose LUTS are related to-or are complicated by-prostatitis, the phenotypical approach to management (e.g., UPOINT) has provided promising results. This method requires further validation and incorporation into clinical practice guidelines. One may also be able to take the lessons learned from this phenotypical approach in prostatitis and apply it to other spheres of LUTS. Recognizing that nocturia is most often caused by pathology outside the urinary tract, urologists will need to develop interdisciplinary networks to encourage other specialties to engage in the management of nocturia to best serve the interests of patients with this systemic problem. As is the case with SUI, the workup of LUTS in men also lacks a solid evidence base to decide what components of assessment are required, which others can be omitted for most patients, and which patients require additional tests, such as cystoscopy, urodynamics, and imaging. Finally, while transurethral resection of the prostate (TURP) remains the gold standard of surgical intervention for $\mathrm{BPH}$, there are other evidence-based interventions (i.e., lasers) that are becoming equally efficacious overall and may be superior for certain subgroups of patients. Clear identification of these subgroups is necessary.

Competing interests: Dr. Herschorn is an ongoing paid consultant with Allergan, American Medical Systems, Pfizer, Promedon, and Johnson and Johnson.

\section{Conclusion}

These are all important questions that remain to be answered. There is no doubt that over time researchers will address these, as well as other dilemmas, and their discussion can form parts of subsequent Canadian Urology Forums.

Correspondence: Dr. Sender Herschorn, Division of Urology, University of Toronto, Sunnybrook Health Sciences Centre, Rm. A309, 2075 Bayview Ave., Toronto ON M4N 3M5; s.herschorn@utoronto.ca 